\title{
Food Industrial Microbial Transglutaminase in Celiac Disease: Treat or Trick
}

\author{
Aaron Lerner, ${ }^{1, *}$, Torsten Matthias ${ }^{2}$ \\ ${ }^{1}$ Pediatric Gastroenterology and Nutrition Unit, Carmel Medical Center, B, Rappaport School of Medicine, Technion-Israel institute of \\ Technology, Haifa, Israel. \\ ${ }^{2}$ Aesku. Kipp Institute, Wendelsheim, Germany \\ *Corresponding author: aaronlerner1948@gmail.com
}

Received March 01, 2015; Revised March 05, 2015; Accepted March 06, 2015

\begin{abstract}
The surge in incidence of celiac disease (CD) is due more to environmental than to genetic changes. It is paralleled by a food industry that is continuously introducing additives to processed products. Microbial transglutaminase (mTG) is an enzyme that deamidates/transamidates proteins, enabling cross-linkage of molecules and revolutionizing the properties of many food products. It belongs to the family of transaminases and tissue transglutaminase (tTG) has been identified as the autoantigen in CD. Both enzymes de/transamidate gluten, the nutritional environmental factor that induces CD. Although several studies have shown that mTG transamidation of wheat flour/gluten can detoxify gluten peptide in vitro, by inhibiting the response to intestinal gliadin, inducing $\mathrm{T}$ cells and reducing INF $\gamma$ production without influencing their main technological properties, a word of caution is advised. The oral challenge of adult CD patients with mTG transamidated flour was found to be only partially effective, not fulfilling expectations, and there have been multiple recent observations that indicate that mTG transamidated flour/gluten could be dangerous to gluten sensitive populations: mTG cross-linking of gluten may be hazardous in CD since the enzyme can deamidate gluten, thus mimicking endogenous tTG, it can link an extensive repertoire of proteins and other macromolecules with immunogenic potential, mTG treated gluten peptides are immunogenic to celiac patients, inducing specific IgA antibodiesand are recognized by gluten-specific humanT cells. Their effect on CD intestinal permeability has not yet been studied. To better understand the actual processes and events, associated with the suggested mTG therapy, long-term ex vivo and in vitro studies are needed. Until then, it is best to respect the data but suspect the safety of the gluten sensitive populations.
\end{abstract}

Keywords: celiac disease, microbial transglutaminase, gluten, immunogenicity, tissue transglutaminase, therapy

Cite This Article: Aaron Lerner, and Torsten Matthias, "Food Industrial Microbial Transglutaminase in Celiac Disease: Treat or Trick.” International Journal of Celiac Disease, vol. 3, no. 1 (2015): 1-6. doi: 10.12691/ijcd-3-1-10.

\section{Introduction}

\subsection{Celiac Disease}

Gluten sensitive enteropathy or celiac disease (CD), is a distinct type of food hypersensitivity defined as an abnormal immunological response to gluten/gliadin which results in a diseased state typically characterized by damage of the lining of the gastrointestinal tract of genetically susceptible individuals. It is the most common type of autoimmune food intolerance in the world and is of life-long duration [1].

Being an autoimmune disease, environmental factors are crucial for disease induction and maintenance. Gluten, which is the storage protein of wheat, and its alcoholsoluble gliadins are the offending inducers of the disease together with structurally related molecules found in barley, rye and oats. However, additional environmental factors, including infections and breast feeding, are associated with CD $[2,3]$. tTG is the autoantigen against which the abnormal immune response is directed [4] and two main autoantibodies, antiendomysium and anti-tTG, provide the currently most useful serological markers to diagnose or screen for the disease [5,6]. Recently, the presence of antibodies to the neo-epitope tTG were shown to be very reliable in the serological diagnosis of the disease $[6,7,8]$. The sequential chain of events operating in the disease was recently unraveled, and gives hope for future therapeutic strategies [9]. Furthermore, the epidemiology, prevalence and clinical presentation of CD are changing constantly and, with time, new clinical presentations are envisaged, increasing the clinical variability of CD $[2,10,11,12]$.

In accordance with its prevalence, the disease may be as much of a concern in developing and emergingcountries as in the developed world. Prevalence rates of $0.3 \%$ and $1 \%$ have been reported in Northern India, and 0.9, 1.2, 1.6 and $0.8 \%$ in Turkish school children, Turkish blood donors, Syrian blood donors and Iranian children, respectively. These prevalence rates are similar to those reported for Europeans and people of European descent (0.4 to $1.2 \%$ ) [13]. Most recently, CD in the Mediterranean 
area was described as an emerging epidemic [14]. In the near future, most new CD cases are expected to come from Africa, Asia and South America. It is estimate that more than 5 million cases will occur in the Mediterranean region in the next 10 years. Without a timely diagnosis and appropriate healthcare, an excess mortality of more than 230,000 cases may be expected overthe next decade [15]. It is estimated that global totals of the number of undiagnosed celiac children have reached 2.2 million (in 2010) of whom 42,000 likely die annually. The estimated number of deaths in children under-the age of 5 attributable to celiac disease was highest in the African region, followed by the Eastern Mediterranean, South-East Asian, Pan-American, European and Western Pacific regions [16].

Gluten is a common ingredient in the modern food industry and cross contamination of products considered or labeled as "gluten-free" is a common problem for gluten sensitive populations. During certain periods in life $50-60 \%$ of the patients do not adhere to their diet, facing the risk of increased morbidity from osteopenia, osteoporosis, nutritional deficiencies, and decreased height during adolescence, secondary autoimmune disorders and gastrointestinal or hematologic malignancies. Thus, the need to eliminate toxic gluten peptides from their food and/or to blunt immunogenic reactions is a wishful task. In fact, numerous dietary and immunologic alternative strategies are in the process of development [9], keeping in mind their safety, effectiveness and affordability.

\subsection{Food Industry Microbial Transglutaminase Applications and Usage}

The extremely high costs of manufacturing transglutaminase from animal origin have prompted scientists to search for new sources of this enzyme. Interdisciplinary efforts have been aimed at producing enzymes synthesized by microorganisms which may have a wider scope of use, resulting in development and production of mTG $[17,18]$. mTG is an enzyme that catalyzes the formation of isopeptide bonds between proteins. Its cross-linking property is widely used in various processes: to manufacture cheese and other dairy products, in meat processing and to manufacture bakery products and prolamine chains. In the bakery, mTG can substantially improve dough elasticity, stability, volume, shelf life and better fermentation tolerance in baked goods such breads, pastas, pastries and tortillas. According to the manufacturers, it is "an excellent element for gluten-free products as well”. Most food proteins, such as caseins, soybean globulins, gluten, actin, myosins, eggs and seafood proteins can be cross-linked by forming an $\varepsilon-(\gamma-$ glutamyl) lysine bond. The enzyme can link even small molecules like histamine, cadaverine, histone etc. as well as epsilon-amines of lysine residues in proteins. The result is an enormous variety and a vast number of new protein complexes with neo-structure, physical and chemical properties and potentially immunogenic neo-epitopes. Additionally, mTG has considerable potential to improve the firmness, flavor, color,bite, texture, viscosity, elasticity and water-binding capacity of food products. Currently, enzymatic preparations are used in almost all industrial branches because of their wide variety and the low costs associated with their biotechnical production processes
[17], and are ingested in large amounts in the common Western diet $[17,18]$. Altogether, a maximum daily intake of mTG could range up to $15 \mathrm{mg}$. Dosing for restructuring is about $50-100 \mathrm{mg}$ of $\mathrm{mTG}$ for each $\mathrm{kg}$ of treated food [19].

The following two paragraphs will bring together the above information on CD and $\mathrm{mTG}$ with respect to therapeutic aspects. The enigma of $\mathrm{mTG}$ as a future therapeutic strategy for CD (treat) will face our current knowledge on the immunogenic/pathogenic properties of the enzyme (trick).

\section{2. mTG as a Potential Therapy for CD- The Treat}

One of the dietary strategies is to detoxify and abolish the immunogenicity of the gluten peptides using various probiotic bacteria or isolated enzymes, be it in the bakery or by oral ingestion. Recently, an Italian group from Avellino, presented an interesting and novel approach to detoxify gluten using TG enzymes [20,21,22,23]. Strangely enough, the authors used tTg, the same CD autoantigen that it is logical to inhibit $[4,9,24]$, in vitro, to decrease the immunogenicity of gluten peptides, thus turning swords into plowshares [25]. tTG mediates transamidation with lysine or lysine methyl ester of the potent immunostimulatory peptide $\alpha$-gliadin $\mathrm{p} 56-68$. The lysine modifiedgliadin peptides, were found to inhibit IFN $\gamma$ production by intestinal T-cell lines derived from HLA-DQ2 positive CD patients, thus suppressing a hallmark cytokine involved in sub-mucosal inflammatory events [20]. Moreover, the authors demonstrated a loss in the response of gliadin-specific intestinal T-cell lines when challenged with gliadin extracted from flour pretreated with mTG (derived from Streptomyces moboraensis) and lysine methyl ester.

In 2012, to challenge their novel potential therapeutic strategy, the same group reintroduced gluten following wheat flour transamidation by mTG, to 47 adult celiac patients on gluten-free diets, in a randomized, controlled clinical study [23]. By day 15 of the study, 37\% of the patients receiving the transamidated flour had relapsed clinically. By day 90, at the end of the study, only16 patients remained free of clinical symptoms, but no variations in IgA-tTG serology, histological biopsies grading and intestinal IFN $\gamma$-mRNA could be found. Despite the partial, in vivo, effectiveness for only a subset of CD patients, the group used a mouse animal model and in vitro intestinal biopsy investigations, to further study the mTG and lysine ethyl ester detoxifying effects of gliadin transamidated peptide [21). They observed that extensive transamidation of wheat flour with lysine ethyl ester by mTG yielded a soluble protein fraction and insoluble transamidated gliadin fraction [21]. By Western blot, they found that these modifications were associated with strongly reduced immune cross-reactivity. With the use of DQ8 tg mice as a model of gluten sensitivity, a dramatic reduction in IFN $\gamma$ production in gliadin-specific spleen cells challenged with soluble protein fraction and insoluble transamidated gliadin fractions, in vitro, was observed. In the soluble protein fraction, they also observed an increase in the IL-10/IFN $\gamma$ protein ratio. In intestinal biopsies from 10 CD patients challenged in vitro 
with gliadins, the authors demonstrated further that insoluble transamidated gliadin dramatically reduced the levels of antigen-specific IFN $\gamma$ mRNA in all specimens responsive to native gliadins. As cytotoxic effects have been described for gliadins, glutathione S-transferase and caspase-3 activities in the enterocytic Caco-2 cell line were investigated. Neither activity was modified by flour transamidation. The results indicate that lysine ethyl ester cross-linking via mTG specifically affects gliadin immunogenicity, reversing the inducible inflammatory response in models of gluten sensitivity without affecting other aspects of the biological activity of gliadins.

In the same year, further delineation of the biochemical modifications of gliadin induced by mTG on wheat flour, was reported [22]. Gliadin and glutenin yields decreased to $7.6 \pm 0.5 \%$ and $7.5 \pm 0.3 \%$, respectively, after a two-step transamidation reaction that produced a water-soluble protein fraction. SDS-PAGE, Western blot and ELISA analyses confirmed the loss of immune cross-reactivity with anti-native gliadin antibodies in residual transamidated gliadins and soluble protein fraction as well as the occurrence of neo-epitopes. Nano-HPLC-ESIMS/MS experiments identified some native and transamidated forms of celiacogenic peptides including p31-49 and confirmed that mTG had similar stereospecificity of tTG. These peptides were found to be $100 \%$ and $57 \%$ modified in the soluble protein fraction and residual transamidated gliadins, respectively. In particular, following transamidation, p31-49 lost its ability to increase tTG activity in Caco-2 cells. Finally, bread manufactured with transamidated flour had only minor changes in baking characteristics. The authors concluded that the two-step transamidation reaction by $\mathrm{mTG}$, modified the analyzed gliadin peptides, which are known to trigger $\mathrm{CD}$, without influencing the main technological properties- thus shedding further light on a detoxification strategy alternative to the gluten-free diet.

Another Italian researcher, this time from Milan, found thatenzymatic modification of gluten by microbial transglutaminase plus lysine prevents the immunologic effect sex pressed by IFN $\gamma$ release, anti endomysium percentage positivity and tTG antibodies levels, transglutaminase activity and immunohistochemical expression of transglutaminase type 2, on cultured duodenal biopsies from celiac patients [26].

A new application formicrobial transglutaminase could be the detoxification of glutenby cross-linking gluten peptides in beverages via isopeptide bonds so that they can be removed by filtration [27].

Several questions, considerations and aspects should be raised, in respect of the use of mTG to inactivate the immunodominant antigenic epitopes in gluten proteins. Some of these were extensively summarized by Schuppan et al. in 2007 [25], but since then, new data has become available.

1. It needs to be shown that mTG processed flour retains the desired texture and baking properties that patients with CD miss in gluten-free products.

2. It would only be effective in controlled settings, such as in places where these modified cereals and flours are used, mainly in developed countries and less so in the underdeveloped ones, where industrial mTG treated food is limited.
3. The cost-effectiveness of large-scale industrial production and costs of these processed flours compared with a gluten-free diet should be calculated.

4. Safety issues regarding the novel products, are of prime importance. The possible generation of neoantigenic epitopes on the cross-linked peptides, needs to be extensively investigated.

5. Another uncertainty regards the as yet ill-defined involvement of innate immunity in the pathogenesis of $\mathrm{CD}$. The innate response is mainly triggered in response to gluten peptides that are different from those peptides that drive adaptive immunity. Innate immunity appears to be independent of gluten deamidation, and even a nearcomplete abrogation of adaptive immunity by gluten transamidation may not be sufficient to suppress celiac genesis.

6. Can complete transamidation of gluten peptides be achieved in flours using the mTG technique, because some patients are very sensitive and react to trace amounts of gluten?

7. The transamidated peptides failed to compete with the native, nondeamidated gluten peptides for binding to HLA-DQ2. Although intestinal T-cell-mediated inflammation in adults appears to be mainly driven by deamidated peptides, this is not the case in infants and young children whose T-cell repertoire is prominently directed against nondeamidated gluten and sequences that are not substrates for tTG.

8. The changes in the nutritional values induced by mTG application on the various proamines are not known.

Below are the observations and arguments against using $\mathrm{mTG}$ in CD.

\section{Food Industrial mTG is a Risk Factor for CD-The Trick}

\section{1. mTG is able to Deamidate Gluten}

A major question arises: Can mTG imitate tTG in deamidating gluten, as is the case in the submucosa of CD patients, thus increasing the immunogenicity of gluten originated toxic peptides? The answer is definitely yes:

Skovbjerg et al studied the deamination and crosslinking of gliadin peptides by different TG. Streptoverticillium transglutaminase, which is used as a cross-linker in the food industry, was able to generate deamidated epitope(s) in a concentration-dependent manner [28]. More recently, a Japanese research group has shown that mTG induced $72 \%$ deamidation when applied to wheat gluten [29]. An upward shift of gluten bands on gel electrophoresis, alteration in secondary structure of the deamidated gluten and deterioration of the aggregation ability of the gluten molecule were reported. mTG has a broader substrate specificity than tTG and deamidates both synthetic and natural gluten peptides [30]. It appears that both TGs, the human and the bacterial ones, are capable of de/transamidation depending on various variables: substrate sequence, affinity and reaction conditions like $\mathrm{pH}$, presence of primary amines and enzyme concentration [17,18,31,32]. Since many studies were performed using synthetic or isolated substrates invitro and since the in-vivo physical/rheological/nutrient composition conditions are much more complicated and 
since both of the TG are sensitive to all these unstable conditions, the ratio of de/transamidation is very sensitive and highly variable. Presently, no data exist on the human luminal or intestinal ratio between tTG and mTG concerning concentration/functionality and gluten handling in the aspect of de/transamidation. In fact, human jejunal TG activity was demonstrated long ago but only tTG was investigated [33].

Gliadin is a good substrate for endogenous and microbial TGs, the specific activities (counts/min/mg) being 9800 and 4290, respectively [34]. Yong YH et al [29], raised the alternative that gliadins are deamidated by microbial or food TGs in the intestinal lumen. A disturbing possibility that TG, in baked products, may act upon gliadin proteins in dough to generate the epitope associated with celiac response, was suggested [35,36]. Even the decreased substrate specificity of mTG, compare to tTG, $[17,18]$ which is an advantage to the food industry, may represent a disadvantage to the celiac population [37].

The ensuing conclusion is that mTG is capable of deamidation of gluten, the degree of which, in vivo, is variable, thus potentially creating immunogenic glutenneo-peptides and enhancing celiac genesis.

\section{2. mTG is Capable of Linking Many Various Molecules to Gluten}

When suitable amino groups are available in the environment, mTG links an amino group to a newly obtained glutamic acid. The amino groups can be small molecules like histamine or cadaverine or epsilon-amines of lysine residues in many proteins (caseins, soybean globulins, gluten, actins, myosins, collagen, egg, fish and meat proteins). This transamidation can cross-link two proteins leading to a complex matrix of proteins bound together. Most recently, mTG was shown, for the first time, to display a much broader acyl-acceptor substrate specificity. Very short-chain alkyl-based amino acids such as glycine and the esterified $\alpha$-amino acids Thr, Ser, Cys, Trp can serve as acceptor substrates [38]. With appropriate primary amines as spacers, various functional groups, carboxyl groups, phosphate groups, saccharides and so on, can be incorporated into protein, including gluten, by using mTG [39]. It is reality, that industry has expanded the use of mTG to include: cross-linking of proteins with agro wastes, production of protein lipidation, modification of hormones by PEGylation, restoration of functionality of gluten from insect-damaged wheat, modification of rheological properties of proteins including wheat, improvement of gelling properties of proteins, emulsification by cross-linking of heterogeneous products originating from the plant and animal kingdoms or the sea, processing of wheat gluten toward biobased or bioplastic material and many more products and applications. The cross-linking of proteins and the capacity to link other nutrient or industrial additives by mTG application, also embeds risk in gluten sensitive populations:

- mTG and proteases have similar substrate preferences for hydrophobic amino acids in the peptide/protein sequence. In addition, glutamine residues attacked by mTG are located in regions characterized by enhanced flexibility, which are also favorable targets for proteases. Finally, mTG has the potential to modify surface proteins, making them more stable against proteolytic digestion
[40]. Based on the above observations, one can envision that mTG can change the natural outcome of gluten digestion in the human intestine, rendering it more resistant to endogenous proteolysis.

- Industrial techniques to improve gluten-free flour/bread using mTG, various extruded flours and protein isolates, were suggested [41,42]. The addition of mTG to the very small quantities of gluten existing in the various gluten-free diets, thus changing various properties of the peptides or promoting protein network formation or new protein complexes, deserves further studyprior to its industrial application.

- The effects of mTG on dough proteins are cultivar dependent. The cultivar Cortazar was found to be the most susceptible. The amounts of $\omega$ and $\alpha+\beta$ gliadins were increased and solubility reduced after treatment with mTG [43]. Changing concentration and solubility of CD toxic peptide will impact their immunogenicity.

- The use of food industrial mTG is expanding, resulting in linking traditional gluten-containing food (like pasta) with gluten-free products. There are numerous examplesof heterologous polymers constructed by mTG usage, but only one will be cited. Fresh yellow alkaline noodles (an oriental noodle) can be cross-linked with soy protein isolate by mTG $[44,45]$. The introduction of traditional gluten-free products to gluten-containing ones, has an unlimited potential toconfuse gluten-sensitive populations. On the shop shelf, when soy is marked on the product, a celiac patient can buy it without paying attention to the second moiety containing gluten. Improvements in food processing technologies do not always conform to the needs of consumer subpopulations. Thereare no limits to such confusion.

\section{3. mTG Treated Gluten Peptides are Immunogenic to Celiac Patients}

- mTG modified gluten proteins were shown to react with celiac sera IgA-anti gliadin antibodies [46].

- mTG-treated cereal prolamines are recognized in an age-related manner by IgA from celiac affected patients [47]. This could reflect a differential manifestation of the effects of such proteins on the intestinal barrier.

- mTG treatment of wheat prolamines in bread increases the serum IgA reactivity of CD patients. Interestingly enough, IgA from pooled celiac sera showed greater reactivity against mTG -treated gluten-free breads than against mTG untreated ones. The electrophoretic pattern of gluten-free bread prolamines was altered by the mTG treatment [48]. The observations imply that mTGtreated breads or gluten-free breads induce immunogenic peptides that react with IgA. This is the appropriate place to be reminded that CD is an IgA mediated disease with specific antibodies against wheat gliadins and tTG.

- mTG deamidated gluten peptides are recognized by gluten-specific $\mathrm{T}$ cells, thus enhancing the immunogenicity of gluten [30].

- Most recently, it was shown that mTG treated gluten peptides applied to cultured intestinal biopsies from CD patients, induced a 15 fold increase in INF $\gamma$ release, and 2.5 and 2.1 fold increases in medium tTG antibody levels and endomysial antibody positivity, respectively [26].

- Contrary to above mentioned celiac patients' immunogenicity toward mTG-treated cereal prolamines 
observations, we demonstrated anti-mTG antibodies in CD patients, the levels of which reflect the degree of their intestinal damage (personal communication).

A study in Switzerland revealed that marketed meat and meat products were found to contain variable amounts of mTG, indicating that mTG used in the food industry finds its way onto the shop shelf oris ingested by consumers, directly to their intestinal lumen [49].

\subsection{Potential Effects of mTG on Intestinal Permeability}

The potential harmful effects of mTG treated food products were recently extensively reviewed [50,51].

Intestinal permeability is increased in CD allowing increased trafficking of macromolecules between the lumen and the host. The tight junction is a complex machinery under strict regulation that acts as a gatekeeper and its abnormal function is a major step in CD pathogenesis [52]. The following are potential mechanisms through which mTG may aggravate the leaky intestine:

- By cross-linking the tight junction proteins to luminal ones, an increased permeability might ensue

- Gluten, by itself, is a well-known inducer of intestinal permeability [52]. Since it is a substrate form TG, neogluten peptides may potentiate the effect of the uncrosslinked molecule.

- mTG cross-links numerous ingredients in the food industry, emulsifying them and facilitating their passage through the tight junction.

- If mTG imitates the protective and trophic functions of tTG on infectious agents and facilitates their luminal survival, the tight junction may leak since infections increase intestinal permeability [52].

\section{Summary}

Despite several studies showing that mTG transamidation of wheat flour/gluten can detoxify gluten peptide in vitro, by inhibiting the response to intestinal gliadin, inducing T-cells and reducing INFy production without influencing their main technological properties, a word of caution is suggested. An oral challenge of adult CD patients with mTG transamidated flour was shown to be partially effective, not fulfilling expectations, and there are multiple recent observations that render mTG transamidated flour/gluten dangerous to gluten sensitive populations. mTG cross-linking of gluten may be hazardous in CD since the enzyme can deamidate gluten, it can link an extended repertoire of protein and other macromolecules with immunogenic potential andmTG treated gluten peptides are immunogenic to celiac patients, whereby their effect on CD intestinal permeability has not yet been checked.

We are not the first to warn against the mTG treat, and many authors have indicated caution for mTG treated wheat/gluten usage in CD [19,28,29,30,35, 36,46,47,48]. Most recently, we have summarized the scientific background and put forward the hypothesis that the increased consumption of food industry bacterial transglutaminase may explain the surge in celiac disease incidence [50]. In addition, we suggested that changes in intestinal tight junction permeability associated with industrial food additives, including mTG, explains the rising incidence of autoimmune disease [51]. More importantly, we recently observed that $\mathrm{mTG}$ is immunogenic in children with $\mathrm{CD}$ and by complexing to gliadin its immunogenicity is enhanced (unpublished, personal communication). In view of the pathogenic role allocated to tTG antibodies, further studies are needed to explore the pathogenic potential of the immunogenic mTG and antimTG antibodies in CD. Improved enzymatic bread making [53, does not guarantee safety to celiac patients.

The final answers to these questions can only be obtained from long-term clinical studies with significant numbers of patients and sensitive readouts, such as cytokine expression measured in intestinal biopsies before and after challenge, mTG activity and functions in the intestinal lumen and wall of celiac patients, immunogenicity of the mTG cross-linked molecules in vivo, competition studies between tTG2 and mTG on ingested intestinal prolamines and the immunopathogenic consequences of mTG treated gluten-free products.

\section{References}

[1] Lerner A, Blank M, Shoenfeld Y. Celiac disease and autoimmunity. Isr J Med Sci. 1996;11:33-36.

[2] Lerner A. Factors affecting the clinical presentation and time diagnosis of celiac disease: The Jerusalem and the West BankGaza experience. Isr J Med Sci. 1994;11:294-295.

[3] Lerner A, Reif S. Celiac disease and infection. In: Infections and Autoimmunity. Eds: Shoenfeld Y and Rose N. 2nd Ed. Elsevier B.V. In press, 2015.

[4] Reif S, Lerner A. Tissue transglutaminase - the key player in celiac disease: a review. Autoimm Rev. 2004;11:40-45.

[5] Lerner A. Serological Diagnosis of Celiac Disease -Moving Beyond the Tip of the Iceberg. Internat $\mathrm{J}$ of Celiac Disease. Editorial. 2014;2:64-66.

[6] Rozenberg O, Lerner A, Pacht A, Grinberg M, Reginashvili D, Henig C, Barak M. A novel algorithm for childhood celiac disease serological diagnosis based upon intestinal biopsies. Crit Rev Allerg Immunol. 2012;11:331-341.

[7] Matthias T, Neidhöfer S, Pfeiffer S, Prager K, Reuter S, Gershwin ME. Novel trends in celiac disease. Cell Mol Immunol. 2011;8:121-5.

[8] Porcelli B, Ferretti F, Vindigni C, Terzuoli L. Assessment of a Test for the Screening and Diagnosis of Celiac Disease. J Clin Lab Anal. 2014 Nov 10. doi: 10.1002/jcla.21816. [Epub ahead of print]

[9] Lerner A. New therapeutic strategies for celiac disease. Autoimmun Rev. 2010;11:144-147.

[10] Lerner A, Agmon-Levin N, Shapira Y, Gilburd B, Reuter S, Lavi I, Shoenfeld Y. The thrombophilic network of autoantibodies in celiac disease. BMC Med. 2013; 11: 89.

[11] Branski D, Ashkenazy A, Frier S, Lerner A, Dinari G, Faber J, Bujanover Y, Jonas A, Lebental E. In: Gluten-Sensitive Enteropathy from Gastrointest Res. Branski D, Rozen P, Kaganoff MF, editor. Basel, Switzerland: Karger; 1992. Extra intestinal manifestation and associated disorders of celiac disease; pp. 164175.

[12] Zelnik N, Pacht A, Obeid R, Lerner A. Range of neurological disorders in patients with celiac disease. Pediatrics. 2004;11:16721676.

[13] Boye JI. Food allergies in developing and emergingeconomies: need for comprehensive data onprevalence rates. Clinical and Translational Allergy 2012, 2:25-33.

[14] Tucci F, Astarita L, Abkari A, Abu-Zekry M, Attard T et al.Celiac disease in the Mediterranean area, BMC Gastroenterology 2014, 14:24-30.

[15] Greco L, Timpone L, Abkari A, Abu-Zekry M, Attard T, Bouguerrà F, Cullufi P,Kansu A, Micetic-Turk D, Mišak Z, Roma E, Shamir R, Terzic S: Burden of celiac disease in the Mediterranean area. World J Gastroenterol 2011, 17: 4971-4978. 
[16] Byass P, Kahn K, Ivarsson A: The global burden of childhood coeliac disease: a neglected component of diarrheal mortality? PLoS One 2011; 6:e22774.

[17] Kieliszek M, Misiewicz A. Microbial transglutaminase and its application in the food industry. A review. Folia Microbiol 2014; 59: 241-250.

[18] Yokoyama K, Nio N, Kikuchi Y. Properties and applications of microbial transglutaminase. Appl Microbiol Biotechnol 2004; 64: 447-454.

[19] Malandain H. Transglutaminases: A meeting point for wheat allergy, celiac disease, and food safety. Europ Ann Aller Clin Immun 2005; 37: 397-403.

[20] Gianfrani C, Siciliano RA, Facchiano AM, Camarca A, Mazzeo MF, Costantini S, Salvati VM, Maurano F, Mazzarella G, Iaquinto G, Bergamo P, Rossi M. Transamidation of wheat flour inhibits the response to gliadin of intestinal $\mathrm{T}$ cells in celiac disease.Gastroenterology. 2007;133:780-9.

[21] Lombardi E1, Bergamo P, Maurano F, Bozzella G, Luongo D, Mazzarella G, Rotondi Aufiero V, Iaquinto G, Rossi M.Selective inhibition of the gliadin-specific, cell-mediated immune response by transamidation with microbial transglutaminase.J Leukoc Biol. 2013 ;93:479-88

[22] Mazzeo MF, Bonavita R, Maurano F, Bergamo P, Siciliano RA, Rossi M. Biochemical modifications of gliadins induced by microbial transglutaminase on wheat flour.Biochim Biophys Acta. 2013;1830:5166-74.

[23] Mazzarella G, Salvati VM, Iaquinto G, Stefanile R, Capobianco F, Luongo D, Bergamo P, Maurano F, Giardullo N, Malamisura B, Rossi M. Reintroduction of gluten following flour transamidation in adult celiac patients: a randomized, controlled clinical study. Clin Dev Immunol. 2012;2012:329150.

[24] Esposito C1, Caputo I, Troncone R. New therapeutic strategies for coeliac disease: tissue transglutaminase as a target. Curr Med Chem. 2007;14:2572-80.

[25] Schuppan D, Junker Y.Turning swords into plowshares: transglutaminase to detoxify gluten. Gastroenterology. 2007;133:1025-8.

[26] Elli L, Roncoroni L, Hils M, Pasternack R, Barisani D, Terrani C, Vaira V, Ferrero S, Bardella MT.Immunological effects of transglutaminase-treated gluten in coeliac disease. Hum Immunol. 2012;73:992-7

[27] Wieser H, Koehler P. Detoxification of gluten by means of enzymatic treatment. J AOAC Int. 2012;95:356-63.

[28] Skovbjerg H, Koch C, Anthonsen D, Sjostrom H. Deamidation and cross-linking of gliadin peptides by transglutaminases and the relation to celiac disease. Biochem Biophys Acta 2004;1690:220230.

[29] Yong YH Yamaguchi S, Matsumura Y. Effects of enzymatic deamidation by protein-glutaminase on structure and functional properties of wheat gluten. J Agric Food Chem 2006;54:60346040.

[30] Dekking EHA, Van Veelen PA, de Ru A et al. Microbial transglutaminase generate $\mathrm{T}$ cell stimulatory epitopes involved in celiac disease. J Cereal Sci. 2008;47:339-346.

[31] Stamnaes J, Fleckenstein B, Sollid LM. The propensity for deamidation and transamidation of peptides by transglutaminase 2 is dependent on substrate affinity and reaction conditions. Biochim Biophys Acta 2008;1784:1804-1811.

[32] Jiang J, Xiong YL. Extreme $\mathrm{pH}$ treatments enhance the structurereinforcement role of soy protein isolate and its emulsions in pork myofibrillar protein gels in the presence of microbial transglutaminase. 2013;93:469-476.

[33] Bruce SE, Bjarnason I, Peters TJ. Human jejunal transglutaminase: demonstration of activity, enzyme kinetics and substrate specificity with special relation to gliadin and coeliac disease. Clin Sci (Lond) 1985;68:573-579.

[34] Skovbjerg H, Noren O, Anthonsen D, Moller J, Sjostrom H. Gliadin is a good substrate of several transglutaminases: possible implication in the pathogenesis of celiac disease. Scand J Gastroenterol 2002;37:812-817.

[35] Gerrard JA, Sutton KH. Addition of transglutaminase to cereal products may generate the epitope responsible for coeliac disease. Trends in Food Sci Technol 2005;16:510-512.

[36] Cottam JRA, Gerrard JA. Protein crosslinking in food-structure, applications, implications for health and food safety. In Food Biochemistry and food processing, 2nd ed. (2012), Chapter 10, pp 207-223. Ed. YH Hui, USA, Blackwell.

[37] Shimba N, Yokoyama K, Suzuki E. NMR-based screening method for tramsglutaminase: rapid analysis of their substrate specificities and reaction rates. J Agric Food Chem 2002;50:1330-1334.

[38] Gundersen MT, Keillor JW, Pelletier JN. Microbial transglutaminase display broad acyl-acceptor substrate specificity. App Microbiol Biotechnol 2013;98:219-230.

[39] Ohtsuka T, Sawa A, Kawabata R, Nio N, Motoki M. Substrate specificities of microbial transglutaminase for primary amines. J Agric Food Chem 2000;48:6230-6233.

[40] Tagami U, Shimba N, Nakaamura M, Yokoyama KI, Suzuki EI, Hirokawa T. Substrate specificity of microbial transglutaminase as revealed by three-dimentional docking simulation and mutagenesis. Protein Eng Des Sel. 2009;22:747-752.

[41] Smerdel B, Pollak L, Novotni D, et al. Improvement of gluten-free bread quality using transglutaminase, various extruded flours and protein isolates. J Food Nutr Res. 2012;51:242-253.

[42] Brenzetti S, Dal Bello F, Arendt EK. Microstructural, fundamental rheology, and baking characteristics of batters and breads from different gluten-free flours treated with a microbial transglutaminase. J Cereal Sci. 2008;48:33-45.

[43] Medina-Rodriguez CL, Torres P, Martinez-Bustos F et al. Effects of microbial transglutaminase on dough proteins of hard and soft (Triticum aestivium) and durum (Triticum durum) wheat cultivars. Cereal Chemist 2009;86:127-132.

[44] Yeoh SY, Alkarkhi AF, Ramli SB, Easa AM. Effect of cooking on physical and sensory properties of fresh yellow alkaline noodles prepared by partial substitution of wheat flour with soy protein isolate and treated with cross-linking agents. Int J Food Sci Nutr 2011;62:410-417.

[45] Aalami M, Leelavathi K. Effect of microbial transglutaminase on spaghetti quality. J Food Sci 2008;73:C306-312.

[46] Berti C, Roncoroni L, Falini ML et al. Celiac-related properties of chemically and enzymatically modified gluten proteins. J Agric Food Chem 2007;55:2482-2488

[47] Cabrera-Chavez F, Rouzaud-Sandez O, Sotelo-Cruz N, Calderon De La Barca AM. Bovine milk caseins and transglutaminasetreated cereal prolamins are differentially recognized by IgA of celiac disease patients according to age. J Agric Food Chem 2009;57:3754-3759.

[48] Cabrera-Chavez F, Rouzaud-Sandez O, Sotelo-Cruz N, Calderon De La Barca AM. Transglutaminase treatment of wheat and maize prolamins of bread increase the serum IgA reactivity of celiac disease patients. Agaric Food Chem 2008;56:1387-1391.

[49] Kaufmann A, Koppel R, Widmer M. Determination of microbial transglutaminase in meat and meat products. Food Addit Contam Part A. Chem Anal Control Expo Risk Assess. 2012;29:1364-1373.

[50] Lerner A, Matthias T. Increased consumption of food industry bacterial transglutaminase may explain the surge in celiac disease incidence. Nutr Reviews, Accepted, 2015.

[51] Lerner A, Matthias T. Changes in intestinal tight junction permeability associated with industrial food additives explain the rising incidence of autoimmune disease. Autoimmun Reviews, In Press, 2015

[52] Fasano A. Zonolin and its regulation of intestinal barrier function: the biological door to inflammation, autoimmunity, and cancer.Physiol Rev. 2011;91:151-175.

[53] Capriles VD, Areas AG. Novel approaches in gluten-free breadmaking: interface between food science, nutrition, and health. Comprhen Rev Food Sci Food Saf 2014;13:871-890. 